\title{
Non-compliance aux messages de prévention de la maladie à virus Ebola en Côte d'ivoire dans un contexte de menace à l'épidémie
} Non-compliance with messages for the prevention of ebola virus disease in Cote d'Ivoire in a context of threats to the epidemic.

Soumahoro SI ${ }^{1,2}$, Kouassi D P ${ }^{1,2}$, Coulibaly $\mathbf{M}^{2}$, Kouamé A D ${ }^{1,2}$, Irika O ${ }^{1,2}$, Ouaga JM ${ }^{1,2}$, Goua Bi DF ${ }^{1,2}$, Yao GHA ${ }^{1,2}$, Angbo-Effi O ${ }^{1,3}, \mathrm{Kra} \mathrm{O}^{1,3}$

${ }^{1}$ Université Alassane Ouattara de Bouaké, ${ }^{2}$ Institut National d'Hygiène Publique, ${ }^{3}$ CHU Bouaké

Correspondant :Dr COULIBALY M'bégnan; Institut National d'Hygiène Publique (INHP) de Bouaké ; Côte d'Ivoire Email : m_begnan@yahoo.fr

\section{Résumé}

Introduction : La pandémie ouest-africaine de 2013-2016 de la maladie à virus Ebola a provoqué une psychose mondiale. Le gouvernement ivoirien, fort de la proximité géographique de la Côte d'Ivoire avec les pays touchés, a instauré plusieurs mesures. Il s'agissait entres autres d'un vaste programme d'éducation sanitaire de la population constitué de 20 mesures à observer par les populations. Nous avons mené cette étude pour apprécier les raisons de non compliance des populations aux mesures de prévention instituées et les déterminants sociodémographiques en rapport.

Méthode: Nous avons mené une étude transversale à visée descriptive et analytique pendant 01 mois, du 11 mars 2017 au 10 avril 2017, dans les ménages de la ville de Bouaké. Nous avons interviewé 405 personnes grâce à un questionnaire. Les logiciels Epi Info 2000 et SPSS 17.0 ont été utilisés pour la saisie et l'analyse statistique des données recueillies.

Résultats : Les adultes avaient une moyenne d'âge

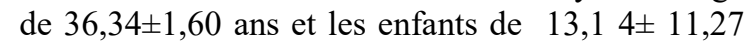
ans. Certains enfants étaient non-scolarisés $(4,3 \%)$ et les adultes avaient un niveau d'instruction supérieur dans $31,3 \%$ des cas. Les mesures les moins observées étaient les mesures $3(42,5 \%), 1$ $(8,6 \%), 5(5,7 \%)$ et $7(5,7 \%)$. Les raisons les plus évoquées étaient la courtoisie $(27,4 \%)$, l'habitude $(21,0 \%)$, le respect de la coutume $(13,1 \%)$ et l'oubli $(11,6 \%)$. Pour la mesure 1, toutes les caractéristiques sociodémographiques y étaient statistiquement associés $(p<0,000)$.

Conclusion :Il importe de cerner tous les contours des messages de sensibilisation et de prévention afin de s'assurer d'une totale adhésion des populations.

Mots-clés : Maladie à virus Ebola - Prévention Communauté - Côte d'Ivoire

\section{Summary}

Introduction: The 2013-2016 West African pandemic of Ebola virus caused a global psychosis. Considering Côte d'Ivoire's geographical proximity with the affected countries, the Ivorian government has introduced several community-based preventive measures. This included a large population health education program consisting of 20 measures to be observed by the population. We conducted this study to assess the reasons for non-compliance of the populations with these preventive measures and socio-demographic determinants related to noncompliance.

Method: We conducted a cross-sectional descriptive and analytical study for 01 month, from March 11, 2017 to April 10, 2017, in the households of the city of Bouaké. We interviewed 405 people through a questionnaire. Epi Info 2000 and SPSS 17.0 software were used for the statistical analysis of the collected data.

Results: Adults had an average age of $36.34 \pm 1.60$ years and children $13.1 \pm 11.27$ years. Some children were out of school (4.3\%) and adults had a higher level of education in $31.3 \%$ of cases. The least observed measures were measures $3(42.5 \%)$, $1(8.6 \%), 5(5.7 \%)$ and $7(5.7 \%)$. The most cited reasons were courtesy $(27.4 \%)$, habit $(21.0 \%)$, respect for custom (13.1\%) and forgetfulness (11.6\%). For measure 1, all socio-demographic characteristics were statistically associated ( $p$ $<0.000$ ).

Conclusion: It's important to identify all the contours of the awareness and prevention messages in order to ensure total population adhesion.

Keywords: Ebola virus disease - Prevention Community - Côte d'Ivoire

\section{INTRODUCTION}

Depuis 40 ans que le virus Ebola a été identifié, l'épidémie ouest-africaine de 2013-2016 qui a sévi en Sierra Léone, au Libéria et en Guinée, reste la plus longue, la plus létale et la plus répandue géographiquement. En Juin 2016, l'Organisation Mondiale de la Santé (OMS) déclarait enfin la fin de l'épidémie avec un total de 28616 cas dont au moins 11310 décès dans l'ensemble de ces pays touchés [1]. Cette épidémie a provoqué une psychose dans les pays touchés et des troubles au sein des populations [2-3]. La Côte d'Ivoire, de par sa proximité avec ces pays sévèrement touchés, était à haut risque d'épidémie [4]. Ainsi, dès le début de la crise, le gouvernement ivoirien a, d'une part, pris des mesures conservatoires pour prévenir l'introduction de la maladie dans le pays, et d'autre part, lancé un vaste programme d'éducation sanitaire de la population portant principalement sur le mode de transmission et les mesures d'éviction de la maladie [5]. La Côte d'Ivoire n'a enregistré aucun cas de maladie à virus Ebola, mais une aucune évaluation des dispositifs et des stratégies 
de préparation à l'épidémie n'a été effectuée, afin de tirer les leçons de cette crise. Nous avons mené cette étude pour évaluer la compliance des populations vis-à-vis des mesures de prévention instituées par le gouvernement, d'en déterminer les raisons de non compliance et les déterminants sociodémographiques en rapport. Cette étude a été menée à Bouaké, deuxième ville de Côte d'Ivoire au plan démographique [6].

\section{POPULATION ET METHODES}

Nous avons mené une étude transversale à visée descriptive et analytique pendant 01 mois, du 11 mars 2017 au 10 avril 2017, dans les ménages de la ville de Bouaké, en milieu urbain. La population d'étude était constituée des membres des ménages. La taille d'échantillon d'étude a été calculée selon la formule de SCHWARTZ :

$\mathrm{N}=\mathrm{t}^{2} \mathrm{p}(1-\mathrm{p}) / \mathrm{i}^{2}$, avec $; \mathrm{N}$ : la taille minimale de l'échantillon nécessaire; $\mathrm{t}$ : l'écart réduit avec un seuil de signification de $5 \% ; \mathrm{p}$ : le pourcentage de ménages supposés avoir de bonnes pratiques en matière d'hygiène ; en l'absence de données, $\mathrm{p}=$ $50 \%$; i : la précision de l'estimation (5\%) ; alors, $\mathrm{N}$ $=(1,96) 2(0,5)(0,5) / 0.052=384$ personnes. Avec un taux de non réponse à $10 \%$, la taille d'échantillon était donc estimée à 422 personnes.

Notre échantillon d'étude a été constitué par un échantillonnage à 3 degrés. Nous avons d'abord réalisé un tirage au sort de deux quartiers à partir du plan des quartiers obtenus auprès du service du cadastre de la Direction des impôts et de 106 ménages dans chacun de ces deux quartiers. Enfin dans chaque ménage, nous avons réalisé un choix raisonné du chef de famille d'une part et d'autre part un enfant âgé d'au moins 10 ans parmi tous ceux de cette même classe d'âge présents était tiré au sort. S'il n'avait pas d'enfant âgé d'au moins 10 ans dans le ménage, seul le chef du ménage était enquêté. Pour l'échantillonnage, deux quartiers de Bouaké ont été tirés au sort pour l'étude, soit, par quartier, 211 personnes. Pour la sélection des membres des ménages, nous avons procédé par un échantillonnage à 4 degrés à partir du plan de ces quartiers obtenus auprès du service du cadastre de la Direction des impôts, Le 1er degré était constitué des deux quartiers tirés au sort. Le 2nd, un secteur géographique du quartier. Le 3ème, les ménages tirés au sort dans le secteur géographique. Pour le 4ème degré, dans les ménages, en plus du chef de famille, un enfant âgé d'au moins 10 ans a été tiré au sort parmi tous ceux de cette même classe d'âge, soit 106 ménages à enquêter. Les sujets à interroger étaient des résidents de Bouaké depuis au moins 6 mois. Les personnes absentes de la Côte d'ivoire pendant la crise de la maladie à virus Ebola ainsi que les enfants incapables de répondre aux questions n'ont pas été incluses. Après consentement éclairé des chefs de ménages, nous avons effectué des interviews individuelles en face à face par nos enquêteurs. Un questionnaire a servi pour les interviews. Pour les personnes mineures, une autorisation verbale a été obtenue de leurs parents (chefs de ménage) avant leur inclusion dans l'étude. Les variables d'étude étaient constituées de variables de caractéristiques sociodémographiques et celles relatives aux mesures de prévention de la maladie à virus Ebola, au nombre de 10 :

- Mesure 1: 'Ne pas chasser ni transporter ni consommer de la viande de brousse",

- Mesure 2 : " $\mathrm{Ne}$ pas ramasser, ni manipuler les animaux morts",

- Mesure 3: " Eviter de se serrer les mains" mains",

Mesure 4 : "Se laver régulièrement les

- Mesure 5 : " En cas de suspicion de cas, éviter tout contact physique avec la personne, l'isoler immédiatement et appeler le centre de santé ou l'hôpital le plus proche ou l'INHP',

- Mesure 6 : " Désinfecter les latrines communes a à l'eau de javel",

Mesure 7 : " Eviter de manipuler les habits des malades et/ou des morts de cause non connue",

- $\quad$ Mesure 8 : " Au cours des rites funéraires, il faut éviter de manipuler le corps du défunt",

- Mesure 9: " $\quad$ Eviter tout contact avec les personnes en provenance des zones en épidémie",

- $\quad$ Mesure 10 : ' Eviter d'effectuer un voyage dans l'un des pays touchés par le virus Ebola',

Pour des considérations éthiques, les questions relatives aux mesures 9 et 10 n'ont pas été posées aux enfants.

Les logiciels Epi Info 2000 et SPSS 17.0 ont été utilisés pour la saisie et l'analyse statistique des données recueillies. Les variables quantitatives ont été décrites par la moyenne et l'écart-type, et les qualitatives par des proportions. Pour l'analyse comparative des données, nous avons utilisé le test $\mathrm{Khi}^{2}$ de Mentel Haenzel et le test de Fisher Exact avec un seuil de signification à 0.05 . Pour des considérations éthiques, les questions relatives aux mesures 9 et 10 n'ont pas été posées aux enfants. Par ailleurs, ce travail n'a pas été soumis à un comité éthique, mais en vertu de la loi sur la pratique des enquêtes, nous avons protégé la confidentialité des renseignements fournis par les personnes interrogées. Par ailleurs, la participation des enquêtés fut libre et obtenue après consentement éclairé et accord verbal. Aucune pression quelle qu'elle soit ne fut faite pour obliger les personnes à participer à l'étude.

\section{RESULTATS}

Caractéristiques sociodémographiques des enquêtés (Tableau I)

Au cours de cette étude, nous avons interrogé 405 personnes dont 214 adultes $(59,51 \%)$ et 164 enfants (40,49\%). Les adultes avaient une moyenne d'âge de 36,34 $\pm 1,60$ ans et les enfants de 13,1 4 $\pm 11,27$ ans. Il y avait une quasi-égalité des sexes chez les 
enfants (sex ratio: 1,02), mais une prédominance du sexe féminin chez les adultes $(52,3 \%$, sexe ratio : 0,92$)$. Les ivoiriens représentaient $88,1 \%$ de l'ensemble des sujets enquêtés, dont 56,8\% enquêtés musulmans et $39,2 \%$, chrétiens. Certains enfants étaient non-scolarisés $(4,3 \%)$ et les adultes avaient un niveau d'instruction supérieur dans $31,3 \%$ des cas.

\section{Raisons de non application des mesures pendant} la crise (Tableau II)

Selon les enquêtés, le non-respect des mesures était dû à la courtoisie $(27,4 \%)$, à l'oubli $(11,6 \%)$, à l'habitude $(21,0 \%)$ et à l'absence de cas de la maladie en Côte d'Ivoire (8,2\%). Parmi les mesures, les moins observées étaient les mesures 3 $(42,5 \%), 1(8,6 \%), 5(5,7 \%)$ et $7(5,7 \%)$. Les raisons les plus évoquées étaient la courtoisie $(27,4 \%)$, l'habitude $(21,0 \%)$, le respect de la coutume $(13,1 \%)$ et l'oubli $(11,6 \%)$. Pour la mesure 1 , étaient avancées comme raisons, l'habitude $(40,0 \%)$ et les raisons économiques $(31,4 \%)$. Les enquêtés évoquaient l'habitude $(21,7 \%)$ comme raisons de non-observance à la mesure 5 , et le respect de la coutume $(67,7 \%)$ pour la mesure 7 . Pour la mesure $8(2,5 \%)$, ils prétendaient l'absence de cas en Côte d'Ivoire $(60,0 \%)$ et le respect de la coutume $(40,0 \%)$.

Déterminants sociodémographiques des personnes refusant l'observation des mesures de prévention

Pour la mesure 1, toutes les caractéristiques sociodémographiques y étaient statistiquement associés $(p<0,000)$; les sujets respectant le moins cette mesure étaient des adultes $(92,9 \%)$, de sexe masculin $(77,1 \%)$, de niveau scolaire supérieur $(42,9 \%)$ et chrétiens $(71,4 \%)$. Pour la mesure 5 , il s'agissait des sujets de sexe masculin $(69,6 \%$, $p=0,041)$. Concernant la mesure 7 , il s'agissait également d'adultes $(80,6 \%, \mathrm{p}=0,013)$, des nonscolarisés $((45,2 \%, p=0,000)$ et les musulmans $(90,3 \%, p<0,001)$. Pour la mesure 9 , ils étaient des non-scolarisés $((46,9 \%, \quad p=0,000)$ et des musulmans $(33,3 \%, p<0,004)$ (Tableau III).

\section{DISCUSSION}

La mesure la moins respectée était la mesure 3 $(42,5 \%)$, relative à l'éviction de se serrer les mains. Pour cette mesure, les sujets de niveau scolaire secondaire et supérieur, et ceux de confessions religieuses chrétienne et musulmane semblaient les plus réfractaires. Vraisemblablement, cette mesure n'épouserait pas certains principes religieux, coutumiers voire moraux, ce qui conduisait à son rejet par des sujets de niveau scolaire plus avancés. Les enquêtés évoquaient la courtoisie $(49,4 \%)$, l'oubli $(22,1 \%)$, l'habitude $(20,3 \%)$ et la sensation d'éloignement du danger en l'absence de cas en Côte d'Ivoire $(8,1 \%)$, comme raison de non compliance à cette mesure. Ne pas se serrer la main lorsqu'on se salue dans le contexte africain pourrait paraitre maladroit. L'habitude avancée comme raison de non application de cette mesure et l'oubli qui en découlait pourraient témoigner de cette norme. Les autorités n'auraient probablement pas tenu compte des us et coutumes des populations avant d'instaurer certaines mesures. A cela, il aurait aussi fallu tenir compte des réalités économiques des populations. Ne pas se conformer à cette disposition paraissait donc justifié. Cette mesure semblait disproportionnée puisque le risque de transmission interhumaine de la maladie semblait obsolète en l'absence de cas notifié en Côte d'Ivoire. Les mesures auraient pu être instituées progressivement par palier de risque, c'est-à-dire des mesures spécifiques en l'absence de cas de la maladie, comme les mesures 1, 2, 9 et 10, puis l'ajout d'autres mesures supplémentaires lorsque des cas seraient notifiés, notamment les mesures 5 , 7 et 8 . Les premières mesures $(1,2,9$ et 10$)$ pourraient être considérées comme mesures contre la contamination initiale, notamment par l'introduction de la maladie en Côte d'Ivoire par les biais des pays touchés par la maladie (Mesures 10 et 9) ou par la transmission de l'animal à l'homme (mesures 1 et 2 ). Les dernières mesures $(5,7$ et 8$)$, auraient été ajoutées pour éviter les transmissions interhumaines et réduire le risque de propagation de la maladie. Les mesures $4,6,7$ et 8 sont par ailleurs des mesures transversales de prévention de maladies transmissibles, mais non spécifiques pour la maladie à virus Ebola, bien que les pratiques culturelles à risque comme la manipulation de malades d'Ebola ou des dépouilles ont fortement contribué à la propagation de la maladie dans les pays touchés [7-9]. Si ces mesures semblaient opportunes pour contrôler de nombreuses maladies transmissibles, leur instauration qu'au stade de la menace en Côte d'Ivoire poserait un problème éthique au regard de l'instauration de mesures non spécifiques de prévention d'une affection bien spécifique (maladie à virus Ebola) au cours d'une période de risque aussi bien spécifique (pas de cas notifié en Côte d'Ivoire). L'absence de prise en compte de ces réalités pourrait s'expliquer par la psychose mondiale occasionnée par cette épidémie [10]. Le grand nombre de mesures de prévention pourrait provoquer la peur au sein de la population comme dans les pays touchés par l'épidémie [11] tout en conduisant à la flambée [2] en cas d'épidémie en Côte d'Ivoire. L'interprétation de ces nombreuses mesures par les communautés pourrait porter à confusion et engendrer la rumeur, si de bonnes stratégies de mobilisation sociale ne sont pas mises en place aux fins de mieux sensibiliser les populations [4]. En effet, la mesure 1 qui devrait contribuer à l'éviction de l'éclosion de l'épidémie en Côte d'Ivoire, en l'absence de cas importés, était pourtant la deuxième mesure la moins respectée. En plus de l'habitude comme raison, les autres étaient d'ordre économique ou liées au fait de l'absence de cas en Côte d'Ivoire. Les populations ne croiraient pas en la possibilité de l'existence de la maladie 
chez nos animaux, pourtant la maladie a déjà été découverte chez l'animal en Côte d'Ivoire [12]. Les raisons économiques liées à l'interdiction de la vente et la consommation de la viande de brousse constitueraient un manque à gagner pour ceux qui sont dans la filière de la viande de brousse. Cette mesure parait aussi difficilement observable pour ceux dont la viande de brousse constituerait une source de protéine gratuite voire bon marché. Cette interdiction de la consommation de la viande de brousse aurait pu être portée sur les animaux chez qui le risque de contamination de la maladie à virus Ebola est plus élevé comme les chiroptères et les primates [9]. Les déterminants retrouvés pour la mesure 1 étaient également observés pour la mesure 3 où les sujets de niveau scolaire secondaire et supérieure, et chrétiens et musulmans refusaient de s'y conformer. Ce constat attesterait des mêmes difficultés d'observation de ces mesures. Pour la mesure la plus respectée (mesure 10), elle le serait de facto du fait que Bouaké n'étant pas frontalière avec les pays en épidémie, les afflux de populations de part et d'autre seraient moindres, d'où l'observation de facto de ces mesures. Cependant la mesure 9 qui pourrait permettre d'éviter la propagation de la maladie en cas d'introduction d'un cas importé pourrait cependant continuer à entretenir la stigmatisation des ressortissants des pays touchés par l'épidémie [3]. Cette mesure n'était d'ailleurs pas respectée pour raison de coutume ou par défiance ou des raisons religieuses. Elle mettrait peut-être à mal les règles d'hospitalité et constituerait des entraves à certains préceptes religieux.

\section{Conclusion}

La crise ouest-africaine de la maladie à virus Ebola, de 2013-2016, a fourni beaucoup d'enseignements quant à la gestion d'un phénomène de santé fortement létal de portée mondiale. Toutefois, il importe de cerner tous les contours des messages de sensibilisation et de prévention afin de s'assurer d'une totale adhésion des populations. Une telle démarche demanderait de la temporisation, de la prise en compte des us et coutumes des populations, afin de produire les mesures les plus adaptées.

Conflit d'intérêt : Aucun.

\section{REFERENCES}

1- World Health Organization. Ebola Virus Situation Report. Geneva, Switzerland: World Health Organization; 2016. http://www.who.int/csr/disease/ebola/situationreports/archive/en/

2- Shultz JM, Cooper JL, Baingana F, Oquendo MA, Espinel Z, Althouse BM, et al. The Role of Fear-Related Behaviors in the 2013-2016 West Africa Ebola Virus Disease Outbreak. Curr Psychiatry Rep. 2016 ; 18 (11): 104
3- Espinola M, Shultz JM, Espinel Z, Althouse BM, Cooper JL, Bainganai F, et al. Fear-related behaviors in situations of mass threat. DISASTER HEALTH.2016 ; 3(4), 102-11.

4- Organisation Mondiale de la Santé (OMS). Préparation de la Côte d'Ivoire face à la maladie à virus Ebola: mission de l'équipe OMS d'appui à la riposte et à la préparation, octobre 2014. Wkly Epidemiol Rec. 2015 ; 90 (8) : 62-6.

5- Gouvernement de Côte d'Ivoire. Communiqué du gouvernement : Mesures préventives face à l'épidémie du virus Ebola. 2014. Consulté le 11 février 2017. Available from :

http://prevention-

ebola.gouv.ci/fichiers/1410955834GOUV\%201etter $\%$ 20EBOLA.pdf.

6- INSTITUT NATIONAL DE STATISTIQUE .Côte d'Ivoire Recensement Général de la Population et de l'Habitat 2014 : Rapport d'exécution et présentation des principaux résultats. $2014 ; 49$ p.

7- Nielsen CF, Kidd S, Sillah ARM, Davis E, Mermin J, Kilmarx PH. Improving Burial Practices and Cemetery Management During an Ebola Virus Disease Epidemic - Sierra Leone, 2014. MMWR . $2015 ; 64(01) ; 20-7$

8- Richards P, Amara J, Ferme MC, Kamara P, Mokuwa E, Sheriff AI, et al. Social Pathways for Ebola Virus Disease in Rural Sierra Leone, and Some Implications for Containment. PLoS Negl Trop Dis.2015;9(4): e0003567.

9- Van Kerkhove MD, Bento AI, Mills HL, Ferguson NM, Donnelly CA. A review of epidemiological parameters from Ebola outbreaks to inform early public health decision-making. Sci Data. 2015; 2: 150019.

10- Shultz JM, Althouse BM, Baingana F, Cooper JL, Espinola M, Greene MC,et al . Fear factor: The unseen perils of the Ebola outbreak. Bull At Sci. 2016 ; 72(5): 304-10.

11- Carrión Martín AI, Derrough T, Honomou P, Kolie N, Diallo B, Koné M,et al. Social and cultural factors behind community resistance during an Ebola outbreak in a village of the Guinean Forest region, February 2015: a field experience. Int Health. 2016 May;8(3):227-9.

12- Le Guenno B, Formenty $\mathbf{P}$, Boesch $\mathbf{C}$. Ebola virus outbreaks in the Ivory Coast and Liberia, 19941995. Curr Top Microbiol Immunol. 1999;235:7784. 
Tableau 1 : Caractéristiques sociodémographiques des populations de Bouaké, 2017

\begin{tabular}{|c|c|c|}
\hline$(n=405)$ & n & $\%$ \\
\hline $\begin{array}{l}\text { Age (ans) } \\
\text { Adultes (18 -87) }\end{array}$ & 241 & 59,5 \\
\hline $\begin{array}{l}\text { Enfants }(10-15) \\
\text { Sexe }(\text { sex ratio }=0,96)\end{array}$ & 164 & 40,5 \\
\hline Masculin & 198 & 48,9 \\
\hline Féminin & 207 & 51,1 \\
\hline Niveau d'instruction & & \\
\hline Aucun & 69 & 17,0 \\
\hline Primaire & 81 & 20,0 \\
\hline Secondaire & 179 & 44,2 \\
\hline Supérieur & 76 & 18,8 \\
\hline Religion & & \\
\hline Chrétienne & 159 & 39,3 \\
\hline Musulmane & 230 & 56,8 \\
\hline Autres & 16 & 3,9 \\
\hline
\end{tabular}


Tableau II : Raisons de non application des mesures de prévention de la maladie à virus Ebola, Bouaké, 2017

\begin{tabular}{|c|c|c|c|c|c|c|c|c|c|c|c|c|c|c|c|c|c|c|c|c|c|c|}
\hline & \multicolumn{2}{|c|}{$\begin{array}{c}\text { Mesure } 1 \\
(n=35)\end{array}$} & \multicolumn{2}{|c|}{$\begin{array}{l}\text { Mesure } 2 \\
(\mathrm{n}=5)\end{array}$} & \multicolumn{2}{|c|}{$\begin{array}{c}\text { Mesure 3 } \\
(\mathrm{n}=172)\end{array}$} & \multicolumn{2}{|c|}{$\begin{array}{c}\text { Mesure } 4 \\
(n=13)\end{array}$} & \multicolumn{2}{|c|}{$\begin{array}{c}\text { Mesure } 5 \\
(n=23)\end{array}$} & \multicolumn{2}{|c|}{$\begin{array}{c}\text { Mesure } 6 \\
(n=4)\end{array}$} & \multicolumn{2}{|c|}{$\begin{array}{c}\text { Mesure } 7 \\
(\mathrm{n}=\mathbf{3 1})\end{array}$} & \multicolumn{2}{|c|}{$\begin{array}{c}\text { Mesure 8 } \\
(n=10)\end{array}$} & \multicolumn{2}{|c|}{$\begin{array}{c}\text { Mesure } 9 \\
(n=32)\end{array}$} & \multicolumn{2}{|c|}{$\begin{array}{c}\text { Mesure } 10 \\
(n=3)\end{array}$} & \multicolumn{2}{|c|}{ Total } \\
\hline & n & $\%$ & $\mathbf{N}$ & $\%$ & $\mathbf{n}$ & $\%$ & $\mathbf{n}$ & $\%$ & $\mathbf{n}$ & $\%$ & $\mathbf{n}$ & $\%$ & $\mathbf{n}$ & $\%$ & $\mathbf{n}$ & $\%$ & $\mathbf{n}$ & $\%$ & $\mathbf{n}$ & $\%$ & $\mathrm{n}$ & $\%$ \\
\hline Habitude & 14 & 40,0 & - & & 35 & 20,3 & 8 & 61,5 & 5 & 21,7 & 4 & 100,0 & 3 & 9,7 & 0 & 0,0 & 0 & 0,0 & 0 & 0,0 & 69 & 21,0 \\
\hline Economique & 11 & 31,4 & - & & - & & - & & 0 & 0,0 & - & & 0 & 0,0 & 0 & 0,0 & 0 & 0,0 & 3 & 100,0 & 14 & 4,3 \\
\hline Religieuse & 3 & 8,6 & - & & 0 & 0,0 & - & & 0 & 0,0 & - & & 0 & 0,0 & 0 & 0,0 & 5 & 15,6 & 0 & 0,0 & 8 & 2,4 \\
\hline Respect de la coutume & - & & - & & 0 & 0,0 & - & & 0 & 0,0 & - & & 21 & 67,7 & 4 & 40,0 & 18 & 56,3 & 0 & 0,0 & 43 & 13,1 \\
\hline Courtoisie & - & & - & & 85 & 49,4 & 5 & 38,5 & 0 & 0,0 & - & & 0 & 0,0 & 0 & 0,0 & 0 & 0,0 & 0 & 0,0 & 90 & 27,4 \\
\hline Oubli & - & & - & & 38 & 22,1 & 0 & 0,0 & 0 & 0,0 & 0 & 0,0 & 0 & 0,0 & 0 & 0,0 & 0 & 0,0 & 0 & 0,0 & 38 & 11,6 \\
\hline $\begin{array}{l}\text { Absence de cas de maladie } \\
\text { à virus Ebola en CI }\end{array}$ & 7 & 20,0 & - & & 14 & 8,1 & 0 & 0,0 & 0 & 0,0 & 0 & 0,0 & 0 & 0,0 & 6 & 60,0 & 0 & 0,0 & 0 & 0,0 & 27 & 8,2 \\
\hline $\begin{array}{l}\text { Disponibilité de la viande } \\
\text { de brousse sur le marché }\end{array}$ & 5 & 14,3 & - & & - & & - & & - & & - & & - & & - & & - & & - & & 5 & 1,5 \\
\hline Assistance à un proche & - & & - & & - & & 7 & 53,8 & & & & & 7 & 22,6 & & 0,0 & & 0,0 & & 0,0 & 14 & 4,3 \\
\hline Défiance & - & & - & & - & & - & & - & & - & & 0 & 0,0 & 0 & 0,0 & 9 & 28,1 & 0 & 0,0 & 9 & 2,7 \\
\hline Total $(n=405)$ & 35 & 8,6 & - & & 172 & 42,5 & 13 & 3,2 & 23 & 5,7 & 4 & 1 & 31 & 5,7 & 10 & 2,5 & 32 & 7,9 & 3 & 0,7 & & \\
\hline
\end{tabular}

- Non applicable 
Tableau III : déterminants sociodémographiques des personnes refusant l'observation des mesures de prévention de la maladie à virus Ebola, Bouaké, 2017

Mesure $1(n=35) \quad$ Mesure $3(n=172) \quad$ Mesure $4(n=13) \quad$ Mesure $5(n=23) \quad$ Mesure $6(n=4) \quad$ Mesure $7(n=31) \quad$ Mesure $8(n=10) \quad$ Mesure $9(n=32)$

\begin{tabular}{|c|c|c|c|c|c|c|c|c|c|c|c|c|c|c|c|c|c|c|}
\hline & IVlesu & $1(n=35)$ & IVlesure & $n=1 / 2)$ & IVlesure & & IVlesur & & IVlesur & $b(n=4)$ & IVlesur & & IVlesur & & & $\theta(n=32)$ & IVlesure & \\
\hline & $\%$ & $p$ & $\%$ & $p$ & $\%$ & $p$ & $\%$ & $p$ & $\%$ & $p$ & $\%$ & $p$ & $\%$ & $p$ & $\%$ & $p$ & $\%$ & $p$ \\
\hline Statut & & 0,003 & & 0,630 & & - & & 0,891 & & - & & 0,013 & & - & & 0,462 & & - \\
\hline Enfant & 17,1 & & 41,9 & & 61,5 & & 39,1 & & 50,0 & & 19,4 & & 10,0 & & 34,4 & & 33,3 & \\
\hline Adulte & 82,9 & & 58,1 & & 38,5 & & 60,9 & & 50,0 & & 80,6 & & 90,0 & & 65,6 & & 66,7 & \\
\hline Sexe & & 0,000 & & 0,558 & & - & & 0,041 & & - & & 0,954 & & - & & 0,812 & & - \\
\hline Masculin & 77,1 & & 50,6 & & 61,5 & & 69,6 & & 75,0 & & 48,4 & & 50,0 & & 46,9 & & 0,0 & \\
\hline Féminin & 22,9 & & 49,4 & & 38,5 & & 30,4 & & 25,0 & & 51,6 & & 50,0 & & 53,1 & & 100 & \\
\hline Niveau d'étude & & 0,001 & & 0,035 & & - & & 0,627 & & - & & 0,000 & & - & & 0,000 & & - \\
\hline Non scolarisé & 5,7 & & 12,2 & & 7,7 & & 21,7 & & 25,0 & & 45,2 & & 5,0 & & 46,9 & & 33,3 & \\
\hline Primaire & 11,4 & & 16,9 & & 15,4 & & 21,7 & & 25,0 & & 29,0 & & 4,0 & & 12,5 & & 33,3 & \\
\hline Secondaire & 40,0 & & 50,00 & & 61,5 & & 47,8 & & 50,0 & & 22,6 & & 1,0 & & 34,4 & & 0,0 & \\
\hline Supérieur & 42,9 & & 20,9 & & 15,4 & & 8,7 & & 0,0 & & 3,2 & & 0,0 & & 6,3 & & 33,3 & \\
\hline Religion & & 0,000 & & 0,000 & & - & & 0,536 & & - & & 0,001 & & - & & 0,004 & & - \\
\hline Chrétienne & 71,4 & & 51,7 & & 69,2 & & 26,1 & & 50,0 & & 6,5 & & 10,0 & & 12,5 & & 66,7 & \\
\hline Musulmanne & 22,9 & & 43,6 & & 30,8 & & 69,6 & & 50,0 & & 90,3 & & 90,0 & & 87,5 & & 33,3 & \\
\hline Autres & 5,7 & & 4,6 & & 0,0 & & 4,3 & & 0,0 & & 3,2 & & 0,0 & & 0,0 & & 0,0 & \\
\hline
\end{tabular}

\begin{tabular}{|c|c|c|c|c|c|c|c|c|c|c|c|c|c|c|c|c|c|c|}
\hline & IVlesu & $1(n=35)$ & IVlesure & $n=1 / 2)$ & IVlesure & & IVlesur & & IVlesur & $b(n=4)$ & IVlesur & & IVlesur & & & $\theta(n=32)$ & IVlesure & \\
\hline & $\%$ & $p$ & $\%$ & $p$ & $\%$ & $p$ & $\%$ & $p$ & $\%$ & $p$ & $\%$ & $p$ & $\%$ & $p$ & $\%$ & $p$ & $\%$ & $p$ \\
\hline Statut & & 0,003 & & 0,630 & & - & & 0,891 & & - & & 0,013 & & - & & 0,462 & & - \\
\hline Enfant & 17,1 & & 41,9 & & 61,5 & & 39,1 & & 50,0 & & 19,4 & & 10,0 & & 34,4 & & 33,3 & \\
\hline Adulte & 82,9 & & 58,1 & & 38,5 & & 60,9 & & 50,0 & & 80,6 & & 90,0 & & 65,6 & & 66,7 & \\
\hline Sexe & & 0,000 & & 0,558 & & - & & 0,041 & & - & & 0,954 & & - & & 0,812 & & - \\
\hline Masculin & 77,1 & & 50,6 & & 61,5 & & 69,6 & & 75,0 & & 48,4 & & 50,0 & & 46,9 & & 0,0 & \\
\hline Féminin & 22,9 & & 49,4 & & 38,5 & & 30,4 & & 25,0 & & 51,6 & & 50,0 & & 53,1 & & 100 & \\
\hline Niveau d'étude & & 0,001 & & 0,035 & & - & & 0,627 & & - & & 0,000 & & - & & 0,000 & & - \\
\hline Non scolarisé & 5,7 & & 12,2 & & 7,7 & & 21,7 & & 25,0 & & 45,2 & & 5,0 & & 46,9 & & 33,3 & \\
\hline Primaire & 11,4 & & 16,9 & & 15,4 & & 21,7 & & 25,0 & & 29,0 & & 4,0 & & 12,5 & & 33,3 & \\
\hline Secondaire & 40,0 & & 50,00 & & 61,5 & & 47,8 & & 50,0 & & 22,6 & & 1,0 & & 34,4 & & 0,0 & \\
\hline Supérieur & 42,9 & & 20,9 & & 15,4 & & 8,7 & & 0,0 & & 3,2 & & 0,0 & & 6,3 & & 33,3 & \\
\hline Religion & & 0,000 & & 0,000 & & - & & 0,536 & & - & & 0,001 & & - & & 0,004 & & - \\
\hline Chrétienne & 71,4 & & 51,7 & & 69,2 & & 26,1 & & 50,0 & & 6,5 & & 10,0 & & 12,5 & & 66,7 & \\
\hline Musulmanne & 22,9 & & 43,6 & & 30,8 & & 69,6 & & 50,0 & & 90,3 & & 90,0 & & 87,5 & & 33,3 & \\
\hline Autres & 5,7 & & 4,6 & & 0,0 & & 4,3 & & 0,0 & & 3,2 & & 0,0 & & 0,0 & & 0,0 & \\
\hline
\end{tabular}

\begin{tabular}{|c|c|c|c|c|c|c|c|c|c|c|c|c|c|c|c|c|c|c|}
\hline & \multicolumn{2}{|c|}{ Mesure 1 (n=35) } & \multicolumn{2}{|c|}{ Mesure 3 ( $n=172)$} & \multicolumn{2}{|c|}{ Mesure 4 (n=13) } & \multicolumn{2}{|c|}{ Mesure 5 (n=23) } & \multicolumn{2}{|c|}{ Mesure $6(n=4)$} & \multicolumn{2}{|c|}{ Mesure 7 (n=31) } & \multicolumn{2}{|c|}{ Mesure $8(n=10)$} & \multicolumn{2}{|c|}{ Mesure 9 (n=32) } & \multicolumn{2}{|c|}{ Mesure $10(n=3)$} \\
\hline & $\%$ & $p$ & $\%$ & $p$ & $\%$ & $p$ & $\%$ & $p$ & $\%$ & $p$ & $\%$ & $p$ & $\%$ & $p$ & $\%$ & $p$ & $\%$ & $p$ \\
\hline Statut & & 0,003 & & 0,630 & & - & & 0,891 & & - & & 0,013 & & - & & 0,462 & & - \\
\hline Enfant & 17,1 & & 41,9 & & 61,5 & & 39,1 & & 50,0 & & 19,4 & & 10,0 & & 34,4 & & 33,3 & \\
\hline Adulte & 82,9 & & 58,1 & & 38,5 & & 60,9 & & 50,0 & & 80,6 & & 90,0 & & 65,6 & & 66,7 & \\
\hline Sexe & & 0,000 & & 0,558 & & - & & 0,041 & & - & & 0,954 & & - & & 0,812 & & - \\
\hline Masculin & 77,1 & & 50,6 & & 61,5 & & 69,6 & & 75,0 & & 48,4 & & 50,0 & & 46,9 & & 0,0 & \\
\hline Féminin & 22,9 & & 49,4 & & 38,5 & & 30,4 & & 25,0 & & 51,6 & & 50,0 & & 53,1 & & 100 & \\
\hline Niveau d'étude & & 0,001 & & 0,035 & & - & & 0,627 & & - & & 0,000 & & - & & 0,000 & & - \\
\hline Non scolarisé & 5,7 & & 12,2 & & 7,7 & & 21,7 & & 25,0 & & 45,2 & & 5,0 & & 46,9 & & 33,3 & \\
\hline Primaire & 11,4 & & 16,9 & & 15,4 & & 21,7 & & 25,0 & & 29,0 & & 4,0 & & 12,5 & & 33,3 & \\
\hline Secondaire & 40,0 & & 50,00 & & 61,5 & & 47,8 & & 50,0 & & 22,6 & & 1,0 & & 34,4 & & 0,0 & \\
\hline Supérieur & 42,9 & & 20,9 & & 15,4 & & 8,7 & & 0,0 & & 3,2 & & 0,0 & & 6,3 & & 33,3 & \\
\hline Religion & & 0,000 & & 0,000 & & - & & 0,536 & & - & & 0,001 & & - & & 0,004 & & - \\
\hline Chrétienne & 71,4 & & 51,7 & & 69,2 & & 26,1 & & 50,0 & & 6,5 & & 10,0 & & 12,5 & & 66,7 & \\
\hline Musulmanne & 22,9 & & 43,6 & & 30,8 & & 69,6 & & 50,0 & & 90,3 & & 90,0 & & 87,5 & & 33,3 & \\
\hline Autres & 5,7 & & 4,6 & & 0,0 & & 4,3 & & 0,0 & & 3,2 & & 0,0 & & 0,0 & & 0,0 & \\
\hline
\end{tabular}

Non applicable 
